\title{
Erratum to: Preserved grip selection planning in chronic unilateral upper extremity amputees
}

\author{
Benjamin A. Philip • Scott H. Frey
}

Published online: 14 November 2013

(C) Springer-Verlag Berlin Heidelberg 2013

\section{Erratum to: Exp Brain Res (2011) 214:437-452 DOI 10.1007/s00221-011-2842-5}

A flawed piece of computer code assigned some trials to the wrong orientation, during the calculation of grip choice likelihoods. These data appear in Figs. 3 and 4, two paragraphs of the Results, and one sentence of the Discussion. (The relevant section of the Discussion is not changed by our revised data, but is included here for completeness.) All other choice/likelihood data reflect averages across orientations, which were not affected by the flaw.

Here are the corrected Results and corrected Figures:

Results (entire "Awkwardness ratings" section)

As shown in Fig. 3 (black lines), a separate group of naïve participants reported minimal awkwardness for thumb placements of $90-120^{\circ}$ and maximum awkwardness for thumb placements of $240-300^{\circ}$. Figure 3 also shows the likelihood of control participants (gray lines) and amputees (dotted lines) selecting each grip (i.e., the choice likelihood) at each orientation. As expected, control and amputee participants were much more likely to choose non-awkward grips in OGS. The correlation between OGS choice

The online version of the original article can be found under doi:10.1007/s00221-011-2842-5.

B. A. Philip $(\bowtie) \cdot$ S. H. Frey

Psychology Department, University of Oregon, Eugene, OR, USA

e-mail: bphilip@uoregon.edu

S. H. Frey

Department of Psychological Sciences, University of Missouri,

Columbia, MO, USA likelihood and awkwardness for controls was -0.857 for the left-hand and -0.886 for the right-hand; for amputees, these correlations reached -0.864 and -0.8932 , respectively $(P<0.001$ for all four). Note that this strong inverse relationship between grip preferences and awkwardness arises even though we calculated the two measures from different populations (choice likelihoods from amputees and matched controls and awkwardness ratings from naïve young participants).

More interestingly, despite the absence of movements, grip preferences in PGS also showed a strong negative correlation with rated awkwardness, as shown by previous work with healthy controls (Johnson 2000a). The correlation between PGS choice likelihood and awkwardness for controls was -0.628 for the left-hand $(P<.05)$ and -0.711 for the right-hand $(P<.01)$; for amputees, these correlations reached $-0.770(P<.01)$ and $-0.768(P<.01)$, respectively. Figure 4 illustrates this by demonstrating the relationship between awkwardness, control choice likelihoods, and amputee choice likelihoods, for the affected hand in the PGS task. Recall that a control participant's "affected" hand was determined by matching with their yoked amputee participant. Notably, Fig. 4 also illustrates the match between grip selections in amputees and controls. Group mean choice likelihoods were nearly identical between the two groups (left-hand $r=0.973, P<0.0001$; right-hand $r=0.993, P<0.0001$ ). This demonstrates that amputees and controls show similar patterns of orientation sensitivity in their grip selections.

Discussion (beginning of "Preserved internal models for the affected hand")

While grip selection tasks generally minimize the use of non-motor strategies (Daprati et al. 2010), amputees could 
Fig. 3 Awkwardness ratings and OGS choice likelihood, for each thumb placement, group mean \pm SEM. All OGS data from intact hand, mirror reversed to produce affected (left) hand data; see text for details. Congruence between high choice likelihood and low awkwardness demonstrates selection of non-awkward grips. a Left-hand. b Right-hand

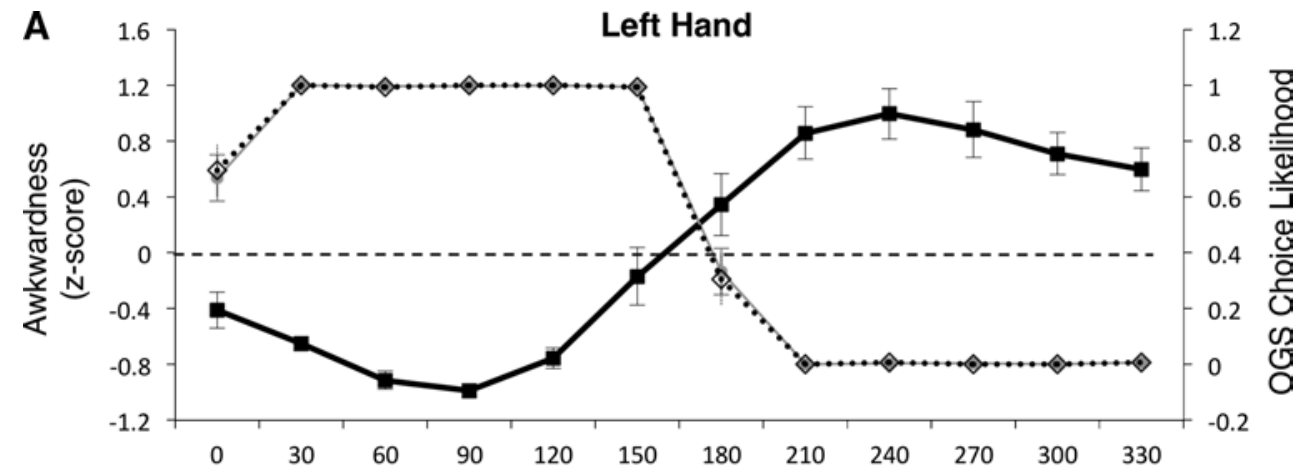

B

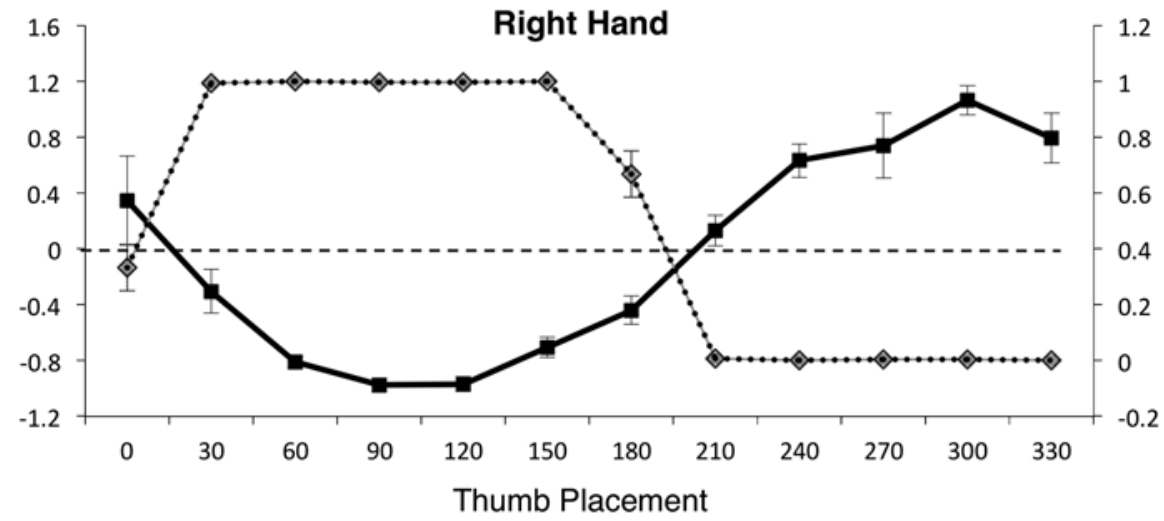

- Awkwardness (z-scored within each participant)
Control Choice Likelihood (0-1 chance of choosing this grip over opposite)
$-1-$ Amputee Choice Likelihood -..-.. Mean $(z=0)$ awkwardness

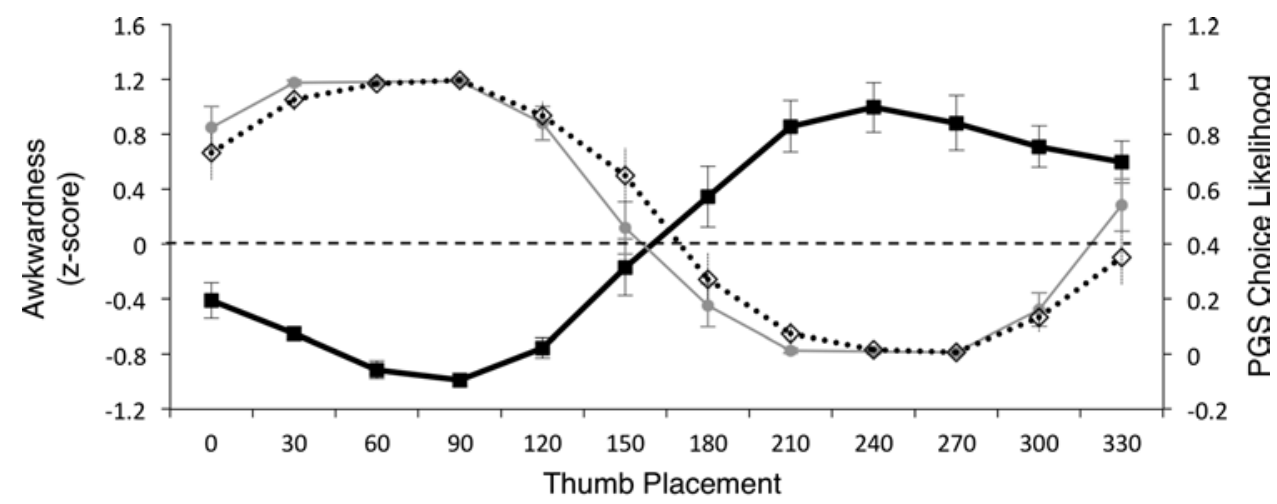

Fig. 4 Relationship between awkwardness ratings and PGS choice likelihood, for affected hand in both amputees and controls. See Fig. 3 for format details use alternative strategies to perform the task based on their affected hand. One such strategy might entail solving the task using visual or cognitive mechanisms. Our data do not support this possibility; amputees and healthy controls show nearly identical patterns of stimulus orientation dependence in PGS (Fig. 4). 